\title{
Diseño de un Centro para Emprendedores en una Escuela Profesional de Ingeniería aplicando el Modelo Lean Canvas
}

\author{
Edilberto Flores-Aguilar \\ Universidad Católica de Santa María, Facultad de Ciencias e Ingenierías Biológicas y Químicas, Escuela Profesional de \\ Ingeniería de Industria Alimentaria, Urb. San José s/n Umacollo, Arequipa, Perú (e-mail: edicato@yahoo.es)
}

Recibido Mar. 5, 2019; Aceptado Abr. 23, 2019; Versión final Jun. 19, 2019, Publicado Dic. 2019

\begin{abstract}
Resumen
El objetivo del presente trabajo fue diseñar un Centro para Emprendedores en una Escuela Profesional de Ingeniería de la Universidad Católica de Santa María (Arequipa, Perú) aplicando el modelo Lean Canvas. Se determinó la intencionalidad emprendedora de los estudiantes, las limitaciones que perciben para crear una empresa y las expectativas de apoyo de la Universidad en la creación de empresas; en el contexto se analizó los factores que influyen en el desarrollo de la actividad emprendedora en la Universidad y se realizó un análisis FODA. El Plan de actividades del Centro demuestra que su implementación es viable. Además, el plan se valida por el impacto en la formación, capacitación de los estudiantes y apoyo en la creación de empresas. Se concluye que la generación de nuevas empresas por los futuros profesionales contribuirá al desarrollo social y económico de la región.
\end{abstract}

Palabras clave: emprendimiento; modelo Lean; diseño; emprendedor; universidad emprendedora

\section{Design of a Center for Entrepreneurs in an Engineering School applying the Lean Canvas Model}

\begin{abstract}
The objective of this work was to design a Center for Entrepreneurs in a Professional School of Engineering of the Catholic University of Santa Maria (Arequipa, Perú) applying the Lean Canvas model. The entrepreneurial intentionality, limitations and expectations that the students perceive to create a company was determined. The factors that influence the development of entrepreneurial activity at the University were analyzed and a SWOT analysis was carried out. The Plan of activities of the Center shows that its implementation is feasible. Also, the plan is validated by the impact on training of students and support in the creation of companies. It is concluded that the generation of new companies by future professionals will contribute to the social and economic development of the region.
\end{abstract}

Keywords: entrepreneurship; lean model; design; entrepreneur; entrepreneurial university 


\section{INTRODUCCION}

Las discusiones relacionadas con políticas ven a las universidades cada vez más como los llamados "motores" de crecimiento económico reconociendo que el impacto económico de las universidades es dependiente, al menos en parte, sobre el éxito de los emprendimientos empresariales afiliados a la universidad (Hayter et al.,2018). En América Latina, se observa que el emprendimiento es un proceso que con el transcurso del tiempo, ha venido causando un impacto positivo en la creación de empresas, fenómeno que coadyuva a la generación de empleos mejorando su eficiencia en la productividad y el logro de un nivel de competitividad en mercados nacionales e internacionales (Sparano, 2014).Según el reporte del Global Entrepreneurship Monitor: Perú 2016-2017, los emprendimientos en el Perú en la etapa temprana se orientan principalmente al sector consumidor. La novedad o innovación en el producto sigue siendo una tarea difícil para los emprendedores y también se evidencia bajo nivel de uso de tecnologías innovadoras originando bajos niveles de productividad. El reporte recomienda mejorar las políticas de gobierno y la educación emprendedora a todo nivel entre otros. (Serida et al., 2017).

Por su lado el Instituto Nacional de Estadística del Perú (INEI) señala que en el año 2017 se dieron de baja un $50.7 \%$ de las empresas creadas. Los emprendedores a la hora de desarrollar una idea o plan de negocios, suelen chocarse fuertemente con la realidad y no se dan cuenta que deben enfrentarse a una serie de factores que quizás no habían previsto: competencia, proveedores, calidad del producto, barreras de entrada. Arroyo (2016) considera al emprendedor como un individuo que goza de cierta libertad y autonomía, proactivo, con actitudes y habilidades que fomentan la innovación, la detección de oportunidades y la asunción controlada de riesgos en situación de incertidumbre y al emprendimiento como "el proceso por el que se detectan nuevas oportunidades y/o se ponen en marcha acciones encaminadas a detectar oportunidades y/o generar nuevas actividades innovadoras, ya sean empresas, productos, procesos, formas de comercialización o formas de gestión pudiendo asumir riesgos controlados en situación de incertidumbre.

Pañoz (2017), señala que el emprendimiento no solo se refiere a la creación de nuevas empresas y/o negocios, hoy en día a su vez es entendida como una competencia que engloba un conjunto de habilidades y destrezas como son la creatividad, el liderazgo, el trabajo en equipo, la innovación, la toma de decisiones, todas ellas demandadas en el ámbito personal, social y profesional que en el fondo implica el desarrollo de una cultura emprendedora.A nivel de la educación superior la formación en emprendimiento está vinculada a los institutos y universidades. Según su desempeño las universidades pueden considerarse en los siguientes tipos: académica, clásica, social, empresarial, emprendedora, en esta ultima el conocimiento se utiliza como un potencial al servicio de los objetivos de su entorno socio económico (Castro y Fernández de Lucio, 2001). En la realidad actual, las universidades no presentan todas este cariz, significa un reto transformarlas para que cumplan ese fin, por otro lado, la inversión estatal en el Perú en materia de investigación base de la innovación, se halla entre las más bajas de nuestro continente $(0,1 \%$ del PBI), en relación con Brasil, México, Colombia o Chile y ni qué decir con los países del primer mundo: Israel está en el 4,7\%, Japón en el 3,8\%, Alemania en el 2,8\% y Estados Unidos en el 2,7\%. (Patrón, 2016).

Hoy la educación superior involucra en el Perú a más de un millón doscientos mil estudiantes en las 142 universidades existentes, entre públicas y privadas, con y sin fines de lucro ubicadas dentro de una realidad caracterizada por la falta de empleo y el desempleo en sus egresados y que alcanzo el año 2012 el $40.2 \%$ de la población económica activa profesional; en este contexto, los egresados tienen la esperanza de que, al culminar sus estudios, puedan encontrar fácilmente un empleo o puedan utilizar sus conocimientos para distintos emprendimientos académicos, empresariales o personales. Sin embargo, esto no siempre es posible porque las universidades e institutos no los preparan necesariamente para acometer estos objetivos, tanto por la desigual calidad de la educación que imparten, como por el tipo de carreras y especialidades que ofrecen además de la falta de empleo ya mencionado. (Gonzales de Olarte, 2016).

Muchas experiencias pueden ser rescatables como la del Tecnológico de Monterrey, la cual ha ido construyendo paulatinamente un ecosistema orientada al emprendimiento, desde los procesos educativos hasta la infraestructura física y los programas de apoyo tecnológico dirigidos a diferentes públicos: sus estudiantes, el empresariado, el sector social y los organismos públicos y privados, alrededor de la tarea de formar emprendedores para el beneficio de su comunidad. (Gonzales y Lopez, 2012). Existen diversos enfoques orientados a fomentar el emprendimiento y al apoyo y desarrollo de empresas: Un enfoque integral del mismo es propuesto por Arroyo-Vázquez, y Van der Sijde (2008), que consideran cuatro ejes: Fomento al emprendimiento, apoyo a los emprendedores, apoyo al desarrollo de empresas, apoyo a la creación de empresas. Vicens y Grullon (2011) propone un modelo considerando tres ejes: 1) Formación del emprendedor, 2) Promoción del valor del emprendimiento e innovación y 3) Desarrollo de un ecosistema de apoyo, el cual debe llevarse de manera integrada. 
En el Perú muy pocas Universidades de las 142 existentes han creado Centros de Emprendimiento, las cuales brindan servicios de capacitación, consultoría y de incubación, sin embargo, se aprecia la carencia de un modelo integral de desarrollo hacia una universidad emprendedora, sin embargo, su presencia puede significar un punto de partida en su dinámica de desarrollo. El diseño de modelos de negocios representa una etapa importante en el lanzamiento de nuevos emprendimientos. El modelo Lean Canvas creado por Ash Maurya, reemplazo algunos bloques del modelo de Ostenwalder con la finalidad de facilitar la detección de los problemas iniciales de todo emprendimiento, los nueve módulos o bloques del modelo están referidos a:

1) Segmento de clientes, 2. Problema, 3. Propuesta única de valor, 4. Solución, 5. Canales, 6. Ingresos, 7. Costes, 8. Métricas clave, 9. Ventaja única o competitiva: (Marchioni, 2013, Ash Maurya, 2012, Osterwalder y Pigneur, 2011).

Las universidades están llamadas a contribuir en el desarrollo social y territorial con nuevos enfoques que promuevan las potencialidades de los estudiantes, el espíritu crítico, la formación de ciudadanos libres y preparados para desenvolverse en situaciones inciertas propias de nuestra época. El desarrollo de nuevos emprendimientos generaría empleo y promovería el crecimiento económico; es necesario en consecuencia formar nuevas promociones con un espíritu emprendedor y con capacidad para llevarla a cabo lo cual aunada a la formación profesional específica potenciaría estas posibilidades.

A medida que la educación superior trabaja para enfrentar los desafíos de la economía cambiante, los programas de ingeniería han aumentado sus programas de emprendimiento como un mecanismo para fomentar las habilidades del siglo XXI en una fuerza laboral diversa (Huang-Saad y Celis, 2017). La esencia de la innovación y la educación para el emprendimiento es un tipo de educación práctica. La implementación del modo de innovación y educación para el emprendimiento en los colegios y universidades puede mejorar el sentido de innovación de los estudiantes, mejorar su calidad integral y aumentar su competitividad en el empleo (Wang et al., 2017). La generación de un Centro para Emprendedores en una Escuela Profesional de Ingeniería sería un punto de partida en la Universidad Católica de Santa María (UCSM) en el nuevo contexto que la realidad exige. La aplicación del modelo Lean Canvas facilitara definir con claridad qué se va ofrecer al mercado, cómo se va realizar, a quién va ser orientado, como se van a vender los servicios y de qué forma se van a generar ingresos, para ello es necesario tener conocimiento previo del entorno, de la intencionalidad de los estudiantes, sus capacidades y expectativas para estructurar un plan de emprendimiento y su viabilidad

\section{METODOLOGIA}

Se desarrolló una metodología orientada a obtener información para el diseño de un centro de emprendimiento en la Escuela Profesional de Ingeniería de Industria Alimentaria (EPIIA) de la UCSM aplicando el modelo Lean Canvas, la metodología se desarrolló en seis pasos: 1) Análisis interno y externo en relación al emprendimiento empresarial, 2) Análisis FODA, 3) Análisis de datos sobre intencionalidad emprendedora, obstáculos y expectativas de los estudiantes obtenidos tras la aplicación de un cuestionario, 4) Diseño del Centro 5) Plan de actividades, 6) Viabilidad de la propuesta.

La búsqueda de la bibliografía se realizó principalmente empleando bases multidisciplinarias con distintos alcances geográficos como la Web of Science (WoS), Scielo (Scientific electronic library online), se efectuó además una búsqueda de información a nivel local tales como marcos legales referidos a la universidad peruana.

A continuación, se describe la metodología empleada:

\section{Análisis interno y externo en relación al emprendimiento empresarial}

Se analizó el Marco legal de la Universidad Peruana, los Estatutos de la Universidad Católica de Santa María (UCSM), su estructura organizativa y las experiencias alcanzadas en relación al emprendimiento universitario. A nivel externo se realizó una revisión sobre el emprendimiento empresarial en el país bajo la óptica del Global Entrepreneurship Monitor (GEM)

\section{Matriz FODA}

Se efectuó un análisis interno (Debilidades y Fortalezas) y externo (Amenazas y Oportunidades) en relación a la creación de un Centro para Emprendedores en la Escuela Profesional de Ingeniería de Industria Alimentaria (EPIIA) de la Universidad Católica de Santa María. 


\section{Intencionalidad emprendedora, obstáculos y expectativas de los estudiantes}

La intención emprendedora de los estudiantes de la EPIIA fue evaluada aplicando un cuestionario empleado en el desarrollo de un trabajo de investigación realizado en la Universidad de Rennes II (Francia), el mismo que se adaptó al presente estudio (Cuestionario sobre intención emprendedora, Université de Rennes II de Francia). La encuesta en el presente estudio fue aplicada en el mes de noviembre del año 2017 a los estudiantes de la EPIIA en los diferentes años académicos: Primer año (II Ciclo), Segundo año (IV Ciclo), Tercer año (VI Ciclo), Cuarto año (VIII Ciclo) y Quinto año (X Ciclo), que se encontraron presentes el día de la encuesta. Las preguntas del cuestionario se indican en la Tabla 1.

En las diversas preguntas excepto la 7,8 y 9 los resultados se expresan considerando el número de estudiantes que marcan la opción seleccionada expresada en porcentaje para cada pregunta con respecto al número de estudiantes encuestados para cada Ciclo de estudios.

En las preguntas 7, 8 y 9 los resultados se expresan multiplicando el número de respuestas dadas por cada ítem con el valor correspondiente en la escala asignada, la suma obtenida por cada ítem se divide entre el número de estudiantes encuestados en el Ciclo correspondiente obteniéndose un valor que refleja la orientación de la respuesta en la escala aplicada. La pregunta 7 considera 20 ítems, la escala aplicada fue de 1 (no se) a 5 (muy importante) para cada ítem, la pregunta 8 considera 23 ítems y la pregunta 9 comprende 11 ítems, en ambas preguntas la escala aplicada fue de 1 (no se) a 5 (totalmente de acuerdo).

Tabla 1: Preguntas del cuestionario aplicado

\begin{tabular}{|c|c|}
\hline Nro. & Pregunta \\
\hline 1 & Datos informativos sobre los estudiantes \\
\hline 2 & Al final de los estudios. ¿Planea crear o asumir el control de una empresa? \\
\hline 3 & ¿Cuándo planea iniciar la creación de su empresa? \\
\hline 4. & ¿Para usted es posible crear su negocio durante sus estudios o inmediatamente después? \\
\hline 5 & ¿Conoces a un empresario dentro de tu familia o entre amigos cercanos? \\
\hline 6 & ¿Tu entorno familiar favorece e incita la creación de empresas? \\
\hline 7 & ¿Explique cuál de estos aspectos son importantes para tu futura vida profesional \\
\hline 8 & ¿Cree usted que las expectativas siguientes serán cubiertas por una carrera de empresario? \\
\hline 9 & $\begin{array}{l}\text { ¿Si tuvieras que iniciar un negocio como estudiante o al finalizar tus estudios? ¿Cuales serían tus principales } \\
\text { obstáculos para que ello se concrete? }\end{array}$ \\
\hline 10 & ¿Alguna vez ha sido informado acerca de los dispositivos de apoyo a las empresas? \\
\hline 11 & ¿Ha buscado información sobre los requisitos exigidos para la creación de empresas? \\
\hline 12 & Conoce de un referente emprendedor en vuestra universidad? \\
\hline 13 & Si dice si, ¿puede usted citar su nombre? \\
\hline 14 & $\begin{array}{l}\text { ¿Conoce de la existencia de una estructura de apoyo a los proyectos de empresas creadas por estudiantes en su } \\
\text { universidad? }\end{array}$ \\
\hline 15 & Piensa usted que la misión de la universidad es: \\
\hline 16 & $\begin{array}{l}\text { ¿Qué tipo de acción le gustaría que su universidad realice en apoyo a la creación de una empresa por los } \\
\text { estudiantes? }\end{array}$ \\
\hline 17 & Qué nivel de conocimiento tiene para cada una de las siguientes organizaciones \\
\hline
\end{tabular}




\section{Diseño del Centro para Emprendedores en la EPIIA.}

Se aplicó el modelo Lean Canvas según la secuencia establecida en sus diversos módulos:

1) Clientes (segmento a ser atendido), 2) Problema, 3) Proposición única de valor, 4) Solución, 5) Canales, 6) Flujo de ingresos, 7) Estructura de costes, 8) Métricas clave, 9) Ventaja competitiva. La Tabla 2 describe el propósito principal de cada módulo

Tabla 2: Propósito de cada módulo en el modelo Lean Canvas

\begin{tabular}{|l|l|}
\hline \multicolumn{1}{|c|}{ Módulo } & \multicolumn{1}{c|}{ Propósito } \\
\hline Segmento clientes & Explica cuál es cliente objetivo sobre el cual hay que trabajar. \\
\hline Problema & $\begin{array}{l}\text { Referido a precisar los tres principales problemas que tienen los clientes objetivo y } \\
\text { determinar cuáles son las soluciones alternativas que usan para resolverlos }\end{array}$ \\
\hline Proposición única de valor & $\begin{array}{l}\text { Sintetiza en una frase de cómo se va ayudar a los clientes para resolver sus } \\
\text { problemas }\end{array}$ \\
\hline Solución & $\begin{array}{l}\text { Se debe precisar las tres características principales del producto/servicio para ayudar } \\
\text { al cliente a resolver sus problemas }\end{array}$ \\
\hline Canales & Define como se va hacer llegar el producto o servicio a los clientes \\
\hline Flujo de Ingresos & Precisa por qué los clientes van a pagar por el producto/servicio \\
\hline Estructura de costes & El análisis de los gastos que se va a tener \\
\hline Métricas Clave & Identificar que actividades clave se va a medir para la toma de decisiones \\
\hline Ventaja competitiva & Refleja en una sola frase lo que hace especial frente al resto de competidores. \\
\hline
\end{tabular}

Las ventajas del Modelo Lean Canvas frente al Plan tradicional es que permite visualizar fácilmente las principales debilidades del proyecto y también sus fortalezas para adoptar las estrategias más convenientes del negocio naciente; al identificar el segmento clientes o los canales para la distribución/difusión se permite conocer mejor el mercado, el Plan de negocio tradicional se recomienda utilizar en una etapa de crecimiento; es decir cuando se ha probado la viabilidad y rentabilidad del nuevo negocio.

\section{Plan de Emprendimiento y Estudio de viabilidad}

Se estableció un Plan de emprendimiento para el primer año de funcionamiento del Centro de emprendedores y que comprende las diversas actividades a desarrollar. También, se realizó el estudio de viabilidad de funcionamiento del Centro de Emprendedores propuesto.

\section{RESULTADOS Y DISCUSION}

A continuación, se presentan los resultados hallados de acuerdo a la metodología presentada

\section{Análisis interno y externo en relación al emprendimiento empresarial}

Las universidades peruanas se rigen por la Ley Universitaria № 30220 promulgada el año 2014 , esta ley contiene muchos aspectos favorables al emprendimiento así, el artículo 52 señala que: "La universidad como parte de su actividad formativa promueve la iniciativa de los estudiantes para la creación de pequeñas y micro empresas de propiedad de los estudiantes, brindando asesoría o facilidades en el uso de los equipos e instalaciones de la institución".

La UCSM, es una universidad particular sin fines de lucro, cuenta con 12 Facultades que involucran a 29 Escuelas Profesionales y una Escuela de Post Grado; con una población estudiantil de aproximadamente 15000 estudiantes. Las Facultades se encuentran ubicadas en cuatro áreas: Ciencias de la salud, Ingenierías, Ciencias Jurídicas y Empresariales y el área de Ciencias Sociales y Humanidades. Los Estatutos de la UCSM en el artículo 5, indica como uno de sus principios la creatividad e innovación para la solución de problemas, la gestión y el emprendimiento; el artículo 79 hace referencia a las incubadoras de empresas. El artículo 97 
señala como atribuciones del Decano de proponer, al Consejo de Facultad, la creación de Escuelas Profesionales, Programas de Segunda Especialidad, Institutos y otros, lo cual posibilita la creación de un Centro de Emprendimiento en la Facultad.

Asimismo, la Visión de la UCSM expresa que la UCSM es una Institución reconocida, por su excelencia; en la formación académica y profesional de personas responsables socialmente; por la producción intelectual e investigación humanística, científica y tecnológica, capaces de contribuir con el desarrollo sostenible.

La Misión considera: a) Formar integralmente líderes profesionales y académicos responsables socialmente, sustentados en principios y valores, con capacidad para insertarse competitivamente en el mundo globalizado, b) Crear, innovar, aplicar, integrar y difundir conocimientos científicos y tecnológicos de las diversas áreas del conocimiento humano, orientados al desarrollo sostenible y c) Fortalecer el capital social en su ámbito de influencia a través de la preservación y promoción del arte, la cultura y el deporte.

En una universidad los aspectos Misión, Gobierno y Estrategia son importantes y conjuntamente con la educación para el emprendimiento, transferencia del conocimiento, intercambio y soporte, internacionalización, compromiso con los stakeholders y que constituyen áreas claves en una universidad emprendedora. (Gibb et al., 2012). En la UCSM los planes estratégicos consideran algunas actividades de promoción al emprendimiento, tales como concursos, ferias de emprendimiento, entre otros. Las Universidades deben de plantearse un reposicionamiento estratégico en base a sus capacidades y considerar dentro de los diversos modelos el enfoque de la cuádruple hélice en la cual el usuario debe de estar en el centro de la relación Gobierno, industria y Universidades y fomentar estas colaboraciones para desarrollar nuevos modelos de negocios a partir de la innovación (Oneill et al., 2017).

El Vicerrectorado de investigación en la UCSM, considera en sus políticas y Planes anuales de Funcionamiento ciertas acciones relacionadas al emprendimiento. En el Plan anual de funcionamiento 2018, se precisa como políticas del Vicerrectorado de Investigación las siguientes: a) Reconocer la investigación, la innovación y el emprendimiento como actividades primordiales de la Universidad. b) Fortalecer la relación Empresa-universidad para el desarrollo de proyectos de investigación, innovación y emprendimiento c) Incentivar el emprendimiento a través de la incubación de ideas de negocios de base tecnológica y de los startups. La EPIIA se encuentra dentro de la Facultad de Ciencias e Ingenierías Biológicas y Químicas; en su formación reciben asignaturas relacionadas a la Ciencia, Tecnología e Ingeniería de alimentos vinculadas a la producción de alimentos lo cual hace pensar la facilidad que tendrían los estudiantes para generar pequeñas y micro empresas. Sin embargo, el currículo actual contiene escazas asignaturas relacionadas a la formación de empresas siendo necesario considerar asignaturas relacionadas con la gestión, marketing, finanzas entre otros.

Como se observa, el marco legal actual posibilita el desarrollo de actividades orientadas a la creación de pequeñas y micro empresas por parte de los estudiantes, la generación de institutos y centros como el que se propone en este trabajo que coadyuvaría el fomento del emprendimiento. La creación de un Centro de Emprendimiento favorecería específicamente las actividades relacionadas a la generación de empresas por parte de los estudiantes, brindaría capacitación y se fomentaría la cultura al emprendimiento en los estudiantes de la escuela, estudiantes de otras facultades y público interesado. A nivel externo las condiciones del ecosistema emprendedor peruano fueron evaluadas por la Encuesta Nacional a expertos (NES) periodo 2016-2017 como parte de la metodología del Global Entrepreneurship Monitor GEM (Serida et al. 2017). Se observa que la educación emprendedora a nivel superior, profesional y universitario ha ido mejorando en su calificación en los años 2015 y 2016, no sucede lo mismo a nivel primario y secundario sin embargo la puntuación alcanzada es baja menos del $50 \%$ de la escala considerada. Así mismo los expertos opinaron acerca de los factores que actualmente apoyan o limitan la actividad emprendedora, considerando como aspectos críticos los relacionados a la transferencia de I+D y las políticas de gobierno, que limitan seriamente el emprendimiento, el primero es muy importante en cuanto a el desarrollo y transferencia de tecnología que promuevan el desarrollo de empresas innovadoras y las políticas de gobierno las cuales deben de orientarse a promover el emprendimiento.

Los expertos elaboraron recomendaciones para promover el emprendimiento en el Perú, dentro de ellas una mejor orientación de las políticas de gobierno, mejorar la educación y capacitación en emprendimiento como aspectos importantes dentro de otros que deben de abordarse de manera integral. El desarrollo de un país emprendedor debe de ser enfocado de manera integral desde el gobierno y las diversas instituciones relacionadas, su desarrollo darían una nueva faz al Perú, consideramos ello como un factor importante para ser más competitivos y de esa forma generar una mayor calidad de vida para sus habitantes. 


\section{Análisis FODA}

Los resultados del análisis interno (Debilidades y Fortalezas) y externo (Amenazas y Oportunidades) de la Escuela Profesional de Ingeniería de Industria Alimentaria (EPIIA) en relación a la creación de un Centro de Emprendimiento expresa lo siguiente:

Fortalezas: La EPIIA dispone de infraestructura, laboratorios y plantas piloto en la cual los estudiantes realizan sus prácticas de las diversas asignaturas consideradas en su currículo en las áreas de ciencia, tecnología e ingeniería de los alimentos, el cual pone énfasis en el desarrollo de nuevos productos, posibilitando la generación de emprendimientos futuros por parte de los estudiantes o egresados. La plana docente de la EPIIA cuenta con experiencia en el campo de la Industria Alimentaria.

Por otro lado, la UCSM brinda diversos servicios a la comunidad, tales como el análisis de alimentos que posibilitaría a los emprendedores en alimentos caracterizar sus productos y cumplir con uno de los requisitos exigidos por el Ministerio de Industria y de Salud, en la inscripción de nuevas empresas o registro de nuevos productos. La UCSM dispone también de recursos humanos en otras Facultades relacionadas al área empresarial como administración, economía, por lo que el Centro que se propone podría interactuar con otras Escuelas Profesionales para llevar adelante sus actividades. La ley Universitaria vigente y los estatutos de la UCSM favorecen la formación emprendedora en los estudiantes y la creación de empresas.

Debilidades: Si bien los estudiantes de la EPIIA reciben una formación en la línea empresarial, este último debe de reforzarse, a través del Centro de Emprendimiento que se propone para brindar una formación emprendedora y apoyo al desarrollo de nuevos emprendimientos, brindar mayor conocimiento en el manejo empresarial, de mercado y financiero para llevar adelante un negocio.

Oportunidades: El campo de la carrera de Industria alimentaria permite generar nuevos productos, fabricarlos y satisfacer necesidades de los consumidores, existe una demanda por más y mejores alimentos y que los estudiantes y/o egresados pueden desarrollar a través de acciones emprendedoras. La población al incrementarse demanda más alimentos, mejores servicios, mejor distribución, precios competitivos, siendo la oferta dirigida a cubrir necesidades internas o de exportación.

Amenazas: Universidades o Escuelas Profesionales poco competitivas pueden quedar relegadas y/o finalmente pueden cerrar por falta de estudiantes, muchos profesionales al terminar su carrera encuentran dificultad para ubicarse laboralmente; brindarles una mejor formación favorecería su competitividad laboral, una formación en el área emprendedora otorgaría mayores posibilidades de éxito dado que pueden formar sus propias empresas o ser captados por empresas emprendedoras que requieren este tipo de profesionales para hacer crecer sus empresas.

\section{Intencionalidad emprendedora, obstáculos y expectativas de los estudiantes}

Población de estudiantes encuestados: De un total de 201 estudiantes matriculados en el año 2017, se encuesto a 145 estudiantes, los cuales se encontraban en sus aulas el día que se realizó la encuesta y que represento el $72 \%$ de la población total. El porcentaje de mujeres en la población de estudiantes encuestados fue mayor al de varones en todos los ciclos académicos: 79, 57, 73, 78 y $66 \%$ de mujeres en los ciclos I, III, V, VII, IX respectivamente. La edad fluctúo entre los 16 a 24 años. Cabe señalar que desde su creación de la EPIIA hace 25 años, siempre mostro la tendencia de tener una población de estudiantes mayor de mujeres que la de varones. Mendoza et al. (2016) al estudiar la iniciativa emprendedora de los estudiantes de una institución de educación superior en el Estado de Hidalgo (México), hallaron que en la carrera de Ingeniería de Industria Alimentaria predomino el número de mujeres (67\%) sobre el número de hombres, situación similar observo en otras carreras tales como Licenciatura en Administración (63.4\%), Ingeniería en Gestión empresarial $(66.9 \%)$.

La Intencionalidad emprendedora se evaluó según los siguientes aspectos: Intención emprendedora para crear una empresa, Entorno del estudiante, Actitud del estudiante sobre la conducta de desempeño futuro como profesional o como empresario. La Intención emprendedora para crear una empresa fue expresada por las respuestas de los estudiantes a las siguientes preguntas ¿Al final de los estudios planea crear o asumir el control de una empresa?, la respuesta fue favorable existiendo un alto porcentaje de estudiantes que responden afirmativamente. Se observa que la intención de "hacerlo definitivamente" decrece a medida que aumenta el nivel de estudios y de "hacerlo probablemente al final de los estudios" aumenta con el nivel de estudios; la suma de las respuestas de ambos sub indicadores refleja un porcentaje alto, más del $90 \%$, los resultados se muestran en la Fig.1. 


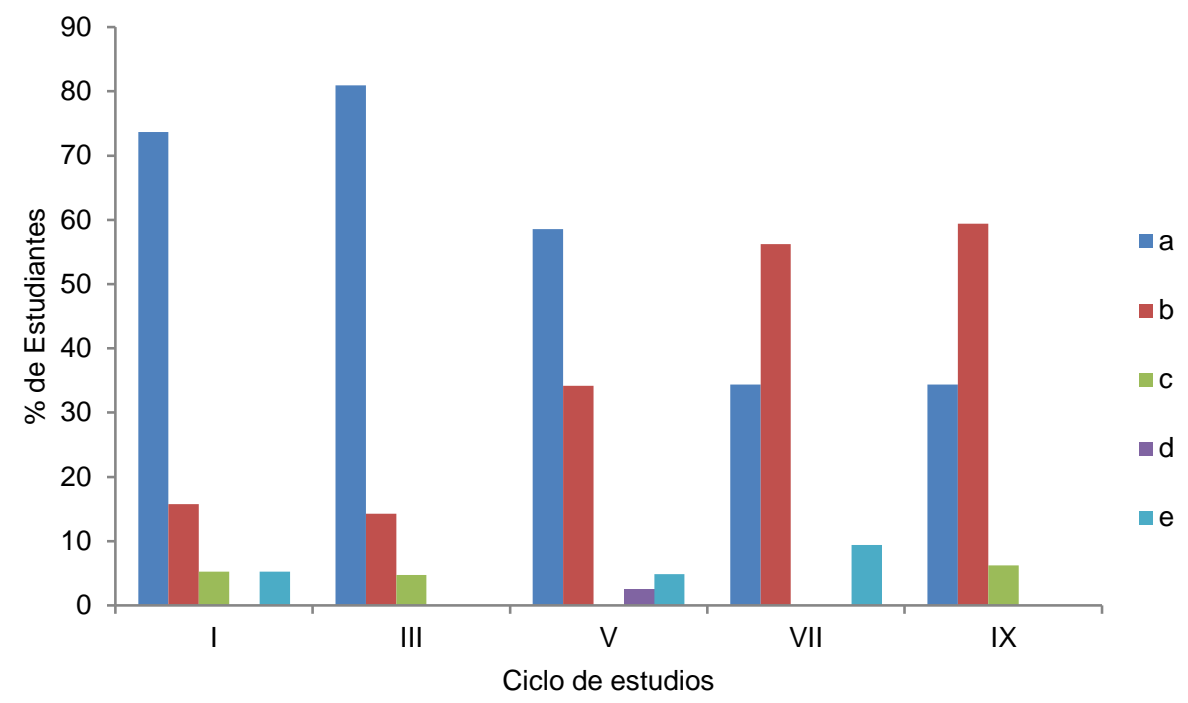

Fig.1: Intención de crear una empresa por los estudiantes al final de sus estudios. a) Si definitivamente, b) Si probablemente, c) No probablemente no, d) No definitivamente no, e) Aun no lo se

A la pregunta ¿Cuándo planea iniciar la creación de su empresa? un porcentaje alto responde favorablemente de efectuarla al final de los estudios o entre unos 3 a 5 años después, según se muestra en la Fig. 2.

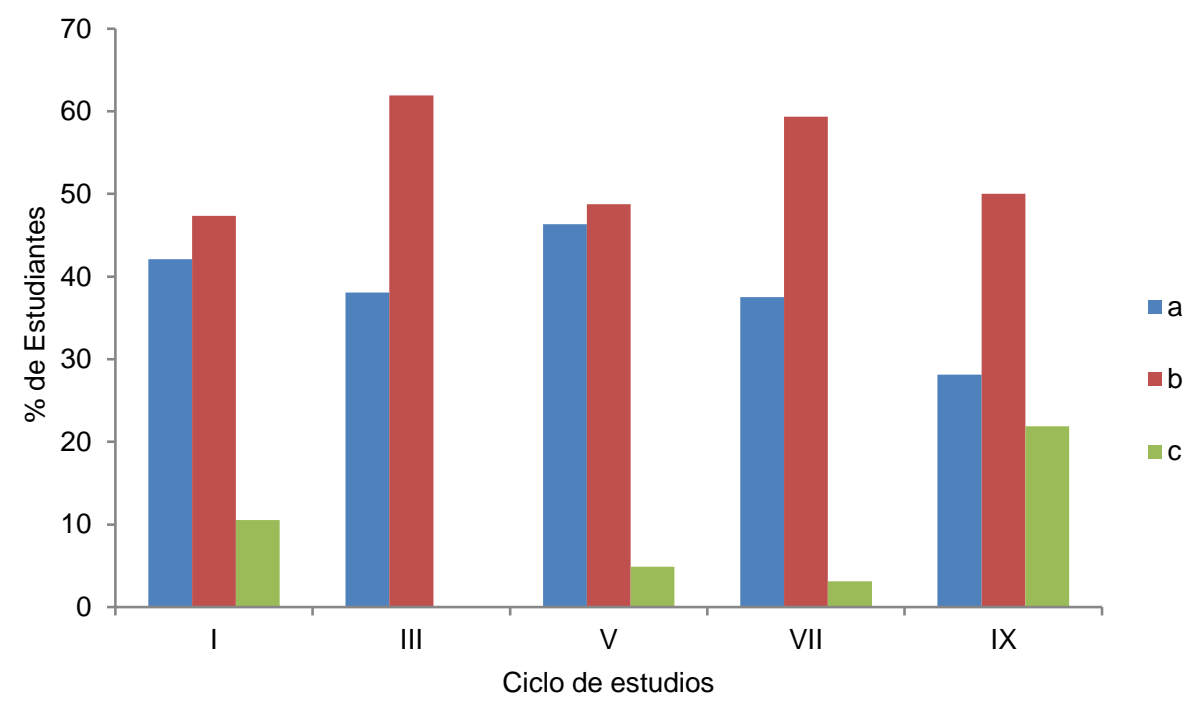

Fig. 2: Cuando planea iniciar la creación de su empresa. a) Durante sus estudios o justo después,

b) 3 a 5 años después de terminar sus estudios, c) Mas de 5 años después de finalizar sus estudios

A la pregunta ¿Para usted es posible crear con facilidad su negocio durante sus estudios o inmediatamente después?, Los estudiantes responden que les resulta muy fácil en un porcentaje que disminuye del $21 \%$ (Ciclo I) al $9 \%$ (Ciclo IX), bastante fácil del 34\% (Ciclo I) al 37\% (Ciclo IX), bastante difícil del 37\% (Ciclo I) al $53 \%$ (Ciclo IX) y muy difícil en un porcentaje reducido, resultados que se aprecian en la Fig. 3.

Los resultados sugieren en los estudiantes un gran potencial emprendedor. La intención de crear una empresa es alta toda vez que se considera como un indicador de emprendedores universitarios potenciales el hecho de emprender en los próximos tres años (Guerrero et al., 2016).

Sobre el entorno del familiar o cercano del estudiante que motivarían la creación de una empresa fue expresada por las respuestas a las siguientes preguntas: ¿Conoces a un empresario dentro de tu familia o entre amigos cercanos? los estudiantes respondieron favorablemente en un porcentaje de $84 \%$ (Ciclo I) y $97 \%$ (Ciclo IX). A la pregunta ¿Tu entorno familiar favorece e incita la creación de empresas?, los estudiantes expresan un entorno favorable a nivel familiar que motiva la creación de empresas, $89 \%$ (I Ciclo), 78 \%(Ciclo IX). Los resultados señalan que estudiantes encuestados presentan un entorno social favorable a nivel de sus padres, familiares y amigos la cual incita a la creación futura de una empresa. 


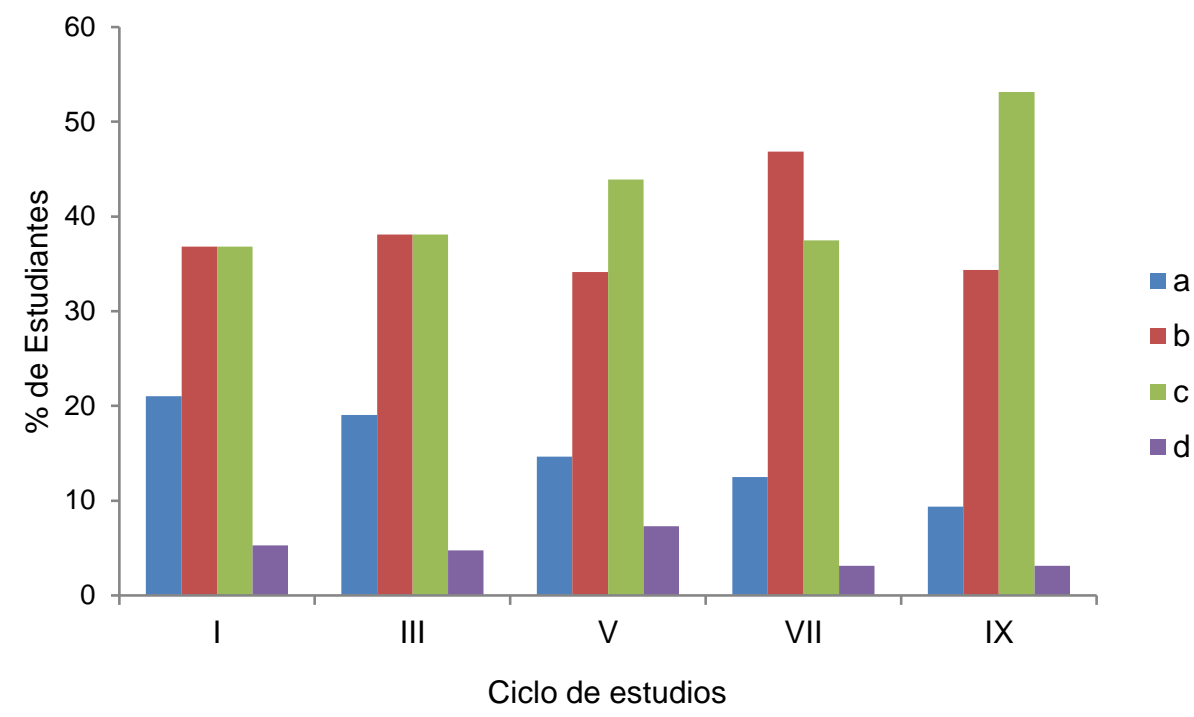

Fig. 3: Posibilidad de crear fácilmente el negocio. a) Si me resulta fácil, b) Es bastante fácil, c) Es bastante difícil, d) Es muy difícil.

El entorno familiar es importante dado que la percepción de los estudiantes al apoyo de su entorno social más cercano, a las opiniones (familia, amigos, compañeros) reflejan la existencia de modelos de referencia asociados al emprendimiento (Reynolds et al., 2005; Veciana et al., 2005; Guerrero y Urbano, 2011) y permite conocer la valoración de cada uno de los elementos que configuran las normas sociales subjetivas, es decir, la influencia de las personas vinculadas al entorno social más cercano en las intenciones emprendedoras (Ajzen, 1991; Liñán y Chen, 2009).

La actitud del estudiante hacia la conducta emprendedora, en su vida profesional futura se evaluó con la siguiente pregunta: ¿Describe cómo estos elementos son importantes para tu vida profesional futura? En la cual se señalan 20 ítems, el estudiante debe calificar para cada ítem según la escala: 1 (no se) a 5 (muy importante). Los estudiantes en sus respuestas priorizaron en primer lugar los siguientes ítems: Tener un ingreso fijo y Ser creativos (promedio 4.7), en segundo lugar: Seguridad en el empleo, Tener un empleo interesante, Tener una expectativa profesional, Tener responsabilidades y estar dispuestos a Enfrentar desafíos (promedio 4.6), en tercer lugar: Ser útiles a la colectividad y Ser vuestro propio jefe (promedio 4.5), cuarto lugar: Ganar mucho dinero, Participar en un proyecto de A- Z (promedio 4.4), quinto lugar: Remuneración en base al empleo, Ser autónomo en el trabajo (promedio 4.3), sexto lugar: Disponer de tiempo libre, Tomar riesgos, Trabajar con otras personas (promedio 4.1), siguen otros ítems con menor puntuación tales como: Tener poder, Pertenecer a un medio social reconocido, No tener un trabajo estresante, Pertenecer a un medio social reconocido.

Al respecto, al determinar las características que configuran el espíritu emprendedor de los creadores de PYMEs en España se identificaron factores generadores y potenciadores, los primeros contienen características esenciales del espíritu emprendedor tales como creatividad, fuerte voluntad, mucha confianza, independencia, en cuanto a los factores potenciadores contienen características que contribuyen a desarrollar el espíritu emprendedor: ambición, espíritu de sacrificio, arriesgado, insatisfacción laboral, entre otros (García del Junco et al., 2007). De igual manera, la actitud del estudiante al asumir a futuro una carrera de empresario fue evaluada en base a la Pregunta ¿Cree usted que las expectativas siguientes serán cubiertas por una carrera de empresario? En la cual se señalan 23 ítems, el estudiante debe calificar de acuerdo a una escala: 1 (no se) a 5 (totalmente de acuerdo).

Los estudiantes priorizaron en primer lugar los siguientes ítems: Tener una perspectiva profesional, Enfrentar desafíos, Realizar sus sueños, Ser creativo, Ser útil a la colectividad, Ser vuestro propio jefe, Tener responsabilidad (promedio 4.5), en Segundo lugar señalaron Tener seguridad en el empleo, trabajar con otras personas, tener un empleo interesante (promedio 4.4), en Tercer lugar: Tener un ingreso fijo, remuneración en base al empleo, participar en proyectos A-Z (promedio 4.3), en Cuarto lugar: Tomar riesgos, ganar mucho dinero, ser autónomos (promedio 4.2). Como se observa la actitud hacia el emprendimiento es favorable según los resultados, dado que permite conocer la valoración de diversos elementos que configuran las actitudes hacia el emprendimiento, según lo señalan también Ajzen (1991) ; Liñán y Chen (2009) y Miranda et al.(2017). Los obstáculos que los estudiantes perciben para crear una empresa fue evaluada con la siguiente pregunta ¿Si tuvieras que iniciar un negocio como estudiante o al finalizar tus estudios? ¿Cuáles serían tus principales obstáculos para que ello se concrete? 
Las respuestas fueron dadas en base a una escala de 1 (no se) a 5 (totalmente de acuerdo), los estudiantes priorizaron sobre 11 ítems los siguientes obstáculos que tendrían que vencer para concretar un negocio, en Primer lugar: Disponer de capital de inicio (promedio 4.6), en segundo lugar: Conocimiento del mercado y Requisitos para crear una empresa (promedio 4.4), en tercer lugar: Ideas innovadoras, Riesgos financieros, La adecuación entre los estudios, la creación de una empresa y los Riesgos financieros (promedio 4.3), en cuarto lugar La experiencia y el Apoyo del entorno (promedio 4.2), en quinto lugar el Contexto de la crisis y la Necesidad de tener de asesores (promedio 4.1), sigue la carga de trabajo (3.9). Los resultados muestran que los estudiantes valoran diversos aspectos que consideran importantes para el desarrollo de una empresa y son conscientes de que la creación de una empresa demanda de conocimiento y de estar capacitados para afrontar el reto y vencer diversos obstáculos que podrían presentarse. Los resultados demandan una mayor capacitación y enfoque al emprendimiento en su formación. En ese sentido, la viabilidad percibida o la percepción sobre la facilidad o dificultad de llegar a ser empresario son denotadas en la encuesta, los estudiantes manifiestan su deseo de crear una empresa, pero perciben diversos obstáculos para concretarlos, resultados similares han sido hallados por Guerrero et al.,(2016) al determinar el perfil emprendedor de estudiantes universitarios españoles.

En cuanto a la información sobre la creación de empresas los estudiantes en un $67 \%$ expresaron que no han recibido información sobre diversas formas de apoyo para crear una empresa (promedio de los cinco años de estudio), sin embargo, se aprecia el interés de los estudiantes en un $57 \%$ de informarse por su cuenta sobre los requisitos para crear una empresa, siendo en mayor grado en estudiantes de años superiores. La mayor parte de los estudiantes desconoce las actividades realizadas por diversos organismos vinculadas al emprendimiento empresarial. Lo anterior denota la necesidad de capacitar en los diversos pasos para crear una empresa tales como: Decisión, La Idea, Plan Empresa, Constitución de la Empresa. (Observatorio Ocupacional, Manual para la creación de empresas por universitarios, 2002). Sobre las expectativas que esperan los estudiantes de la Universidad, los estudiantes respondieron de la siguiente manera: a las preguntas: ¿Conoce de la existencia de una estructura de apoyo a los proyectos de empresas creadas por estudiantes en su Universidad? los estudiantes en un $87 \%$ señalaron desconocer la existencia de una estructura de apoyo por parte de la universidad a proyectos de creación de empresas creadas por estudiantes. Con respecto a la misión de la Universidad señalaron que esta debe: Formar estudiantes para la creación de empresas (promedio 82\%), acompañar al estudiante en la creación de empresas (promedio 48\%), de informar sobre la creación de empresas (promedio 68\%),

En relación a la pregunta ¿Qué tipo de acción le gustaría que su universidad realice en apoyo de la creación de una empresa por los estudiantes? los estudiantes señalaron las siguientes acciones: a) Desarrollar programas dirigidos a jóvenes para sacar adelante un proyecto innovador (promedio 54\%), b) Apoyo para crear modelos de negocios y asesoría personalizada (promedio 26\%), c) Organización de talleres y diplomados (promedio 14\%) y d) Incubación de proyectos (promedio 6\%). Finalmente, los estudiantes señalaron que no conocen ningún referente emprendedor en la universidad (promedio $82 \%$ ). La problemática para generar una empresa, por lo general tiene relación con la falta de información en cómo desarrollar una idea de negocio, las formas jurídicas más recomendables para su idea, así como la falta de financiación o la existencia de subvenciones; por otro lado, la alta mortandad de las empresas en los primeros años de vida es un problema fundamental y que debe superarse con una buena formación en creación y dirección de empresas (Aguado, 2008). Para una universidad clásica, Liventsova y Malkova (2017) señalan que para desarrollar competencias sociales y empresariales la institución debería proporcionar, junto con otras condiciones, una estructura para que los estudiantes se muevan a través de todas las etapas: obtención de experiencia en la solución de problemas sociales, la experiencia en el desarrollo y la implementación de proyectos sociales reales y de reflexión empresarial. Para este propósito, la creación de centros especiales orientados a ese fin resulta importante.

\section{Diseño del Centro para Emprendedores aplicando el Modelo Lean Canvas}

El Centro para Emprendedores que se propone permitirá paliar esta demanda en la formación y apoyo al emprendimiento de los estudiantes; para el diseño del mismo se consideran los nueve módulos o bloques siguientes de acuerdo al Modelo Lean Canvas, como se muestra en la Tabla 3. 
Tabla 3: El Modelo Lean Canvas orientado al diseño del Centro de Emprendedores de la EPIIA.

\begin{tabular}{|c|c|}
\hline Segmento clientes: & $\begin{array}{l}\text { El mercado objetivo en la presente propuesta son los estudiantes de la EPIIA y estudiantes de } \\
\text { otras Facultades de la propia universidad y público interesado. }\end{array}$ \\
\hline \multirow{7}{*}{ Problema: } & $\begin{array}{l}\text { La encuesta aplicada a los estudiantes de la EPIIA permitió no solo determinar su intención } \\
\text { emprendedora sino conocer sus necesidades los cuales se tomaron en cuenta en el diseño del } \\
\text { Centro para Emprendedores que se propone. Se priorizaron tres problemas de acuerdo a la } \\
\text { metodología del Lean Canvas: }\end{array}$ \\
\hline & $\begin{array}{l}\text { Problema 1: Falta de una estructura organizacional que apoye el emprendimiento en sus } \\
\text { estudiantes para la creación de empresas en la EPIIA. }\end{array}$ \\
\hline & $\begin{array}{l}\text { Solución: La creación de un Centro para Emprendedores en la EPIIA permitirá estructurar, } \\
\text { dinamizar acciones de formación, apoyo y desarrollo de empresas. }\end{array}$ \\
\hline & $\begin{array}{l}\text { Problema 2: La formación de los estudiantes en emprendimiento para la creación de empresas } \\
\text { no es desarrollada por la EPIIA como debería esperarse. }\end{array}$ \\
\hline & $\begin{array}{l}\text { Solución: Propiciar la formación de estudiantes en emprendimiento para la creación de } \\
\text { empresas a partir de una estructura diseñada que funcione como un Centro para fomentar el } \\
\text { emprendimiento. }\end{array}$ \\
\hline & Problema 3: Falta de programas dirigidos a jóvenes para sacar adelante un proyecto innovador. \\
\hline & $\begin{array}{l}\text { Solución: El Centro para emprendedores que se propone, organizara dentro sus actividades } \\
\text { Programas orientados a la formación, apoyo y generación de empresas por los estudiantes de } \\
\text { la EPIIA. }\end{array}$ \\
\hline $\begin{array}{l}\text { Propuesta única de } \\
\text { valor: }\end{array}$ & $\begin{array}{l}\text { La propuesta única de valor se expresa como sigue: "Crear e innovar para el desarrollo a través } \\
\text { del emprendimiento empresarial" }\end{array}$ \\
\hline Solución: & $\begin{array}{l}\text { El Centro para emprendedores de la Escuela Profesional de Ingeniería de Industria Alimentaria } \\
\text { desarrollara sus actividades brindando los siguientes servicios: a) Organización de cursos de } \\
\text { creatividad y emprendimiento b) Desarrollo de Programas de capacitación y entrenamiento para } \\
\text { emprendedores c) Desarrollo de Programas de apoyo en la creación de empresas que } \\
\text { comprendan actividades de pre incubación, incubación y seguimiento. }\end{array}$ \\
\hline Canales: & $\begin{array}{l}\text { Para llegar a los clientes se realizara la difusión de las actividades del centro a través de una } \\
\text { página virtual a desarrollar por el Centro para emprendedores propuesto y de la propia } \\
\text { universidad, se realizara adicionalmente la difusión de actividades por medio de la prensa }\end{array}$ \\
\hline Ingresos: & $\begin{array}{l}\text { Los ingresos del centro se generarán principalmente por el pago por los servicios prestados a } \\
\text { los estudiantes y público interesado en el mismo. En tal sentido El centro desarrollara diversos } \\
\text { programas de fomento y apoyo al emprendedor para la creación de sus propias empresas. }\end{array}$ \\
\hline Costos: & $\begin{array}{l}\text { Los costos están referidos al pago de docentes y personal involucrado en los servicios } \\
\text { prestados, así como gastos de publicidad. }\end{array}$ \\
\hline Métricas clave: & $\begin{array}{l}\text { Consideramos de acuerdo a Marchioni (2013), las siguientes métricas a tomar en cuenta: } \\
\text { a) Tasa de activación de clientes que han ingresado en la página web, b) Coeficiente de } \\
\text { rentabilidad, tales como la eficiencia operativa a partir de la optimización de los costos directos } \\
\text { del producto o servicio ofrecido, c) Costos de adquisición del cliente }\end{array}$ \\
\hline $\begin{array}{l}\text { Ventaja única o } \\
\text { competitiva: }\end{array}$ & $\begin{array}{l}\text { Principalmente la formación de emprendedores universitarios y apoyo al emprendedor a crear } \\
\text { su propia empresa. }\end{array}$ \\
\hline
\end{tabular}

\section{Plan de funcionamiento del Centro de Emprendedores}

Los resultados hallados en este trabajo, así como la priorización dada de acuerdo al modelo Lean Canvas permiten establecer el siguiente Plan de funcionamiento y presentado en este trabajo de manera resumida para el primer año de funcionamiento, como se muestra en la Tabla 4. 
Tabla 4: Resumen Plan de Funcionamiento del Centro para su Primer año.

\begin{tabular}{|c|c|}
\hline Servicios a ofertar: & $\begin{array}{l}\text { Están dentro de las siguientes acciones: a) Programa de Cursos para fomentar la } \\
\text { creatividad y el emprendimiento, b) Programas de capacitación y entrenamiento para } \\
\text { emprendedores, c) Programas de apoyo en la creación de empresas: Pre incubación, } \\
\text { Incubación y Seguimiento, d) Apoyo o asesoría a emprendedores en el aspecto } \\
\text { empresarial, e) Organización de Concursos de emprendimientos para Identificar el } \\
\text { talento emprendedor y futuros negocios con alto potencial de crecimiento e innovación. }\end{array}$ \\
\hline Usuarios: & $\begin{array}{l}\text { Dirigido a los estudiantes de la Escuela profesional de Ingeniería de Industria } \\
\text { Alimentaria, estudiantes de otras Facultades de la Universidad y Público en general. }\end{array}$ \\
\hline $\begin{array}{l}\text { Tecnologías requeridas y } \\
\text { su Disponibilidad:. }\end{array}$ & $\begin{array}{l}\text { Plataforma virtual, laboratorios, plantas piloto, aulas, oficinas, los mismos que se } \\
\text { encuentran disponibles por la Facultad. }\end{array}$ \\
\hline Precio: & Son definidos de acuerdo al tipo de servicio que se preste. \\
\hline $\begin{array}{l}\text { Organización, Personal y } \\
\text { Recursos: }\end{array}$ & $\begin{array}{l}\text { El centro se organizara con tres departamentos: Promoción, capacitación y } \\
\text { asesoramiento a través de las cuales se organizara el desarrollo de las diversas } \\
\text { actividades programadas. }\end{array}$ \\
\hline Personal requerido: & $\begin{array}{l}\text { Un coordinador general: responsable de la gestión del proyecto y de presentar los } \\
\text { informes de avance técnicos y financieros, Un coordinador administrativo: encargado de } \\
\text { la rendición de gastos del proyecto, Personal profesional y/o técnico conformado por } \\
\text { docentes de la universidad, así como por personal complementario contratado de } \\
\text { acuerdo a la naturaleza de las actividades programadas. }\end{array}$ \\
\hline Recursos implicados: & $\begin{array}{l}\text { Se requiere de una oficina de coordinación, auditorios o ambientes para el desarrollo de } \\
\text { actividades de difusión, laboratorios de Industria alimentaria y de equipamiento piloto, } \\
\text { plataforma virtual, servicios de Internet, página web. }\end{array}$ \\
\hline Plan de actividades: & $\begin{array}{l}\text { Se considera el Desarrollo de cursos de creatividad y emprendimiento: } \\
\text { - Incrementando mi creatividad } \\
\text { - } \quad \text { Emprendimiento e innovación } \\
\text { - } \quad \text { Gestión Empresarial } \\
\text { - Marketing Empresarial } \\
\text { Asesoría a emprendedores: } \\
\text { - Dirigido a estudiantes de pregrado y egresados } \\
\text { - Dirigido a público interesado } \\
\text { Cursos de capacitación y entrenamiento para emprendedores: } \\
\text { - Desarrollo de nuevos productos } \\
\text { - Como crear un negocio } \\
\text { Concursos de innovación y emprendimiento. }\end{array}$ \\
\hline $\begin{array}{l}\text { Presupuesto de ingresos y } \\
\text { gastos: }\end{array}$ & $\begin{array}{l}\text { Los gastos previstos consideran el pago por servicios profesionales en el desarrollo de } \\
\text { cursos, asesorías de acuerdo a una programación anual de actividades, gastos de } \\
\text { gestión, gastos de publicidad y de oficina. Los ingresos previstos resultan del pago de } \\
\text { los usuarios por los servicios prestados en las diversas actividades programadas. }\end{array}$ \\
\hline Saldo: & Considera la diferencia entre los ingresos y gastos efectuados \\
\hline
\end{tabular}

\section{Estudio de viabilidad}

El diseño del Centro plantea un sistema organizativo flexible, el cual considera tres Departamentos: Promoción, Capacitación y de Servicios. El Plan de funcionamiento del Centro de Emprendimiento considera diversas actividades orientadas al fomento de la creatividad y del emprendimiento a través del desarrollo de cursos presenciales, realización de concursos para identificar al talento emprendedor y desarrollo de cursos de entrenamiento más específicos y necesarios en la capacitación del emprendedor, haciendo más 
competitivos a los estudiantes y egresados y posibilitar la formación de nuevas empresas. Los estudiantes requieren una formación que propicie una cultura emprendedora, promuevan el desarrollo de sus propias empresas como una alternativa de desarrollo profesional ante la falta de empleo, además el Sector empresarial en la región requiere del concurso de la Universidad a través de sus diversas Facultades para promover el emprendimiento empresarial, el aspecto de innovación es importante en las empresas en funcionamiento y en las nuevas empresas que se formen, la Universidad puede contribuir a dar ese salto tecnológico requerido formando profesionales con espíritu crítico generando nuevas alternativas de desarrollo a través del emprendimiento propiciando además una sociedad más equitativa.

Los servicios que ofrecerá el Centro para Emprendedores están orientados a los estudiantes de la EPIIA y de la Universidad además de público en general y comunidad empresarial. Las actividades a desarrollarse, así como los servicios que ofrecerá el Centro serán difundidas a través de la página web de la Universidad y por otros medios de difusión. La naturaleza del proyecto permitirá generar ingresos por las actividades a desarrollar las cuales pretenden, propiciar el desarrollo de la creatividad y del emprendimiento, estimular al talento emprendedor, apoyo al desarrollo empresarial de la región lo cual implica mayores ingresos a la economía y un aumento en la creación de puestos de trabajo.

La evaluación financiera de los gastos con respecto a los ingresos previstos genera un saldo positivo en el primer año, el proyecto resulta rentable económicamente, considerando el desarrollo del Plan de funcionamiento del Centro, el desarrollo de sus diversas actividades programadas en su primer año originaria un saldo favorable de S/. 9800.00 soles si a ello se suman los beneficios sociales inclinaría la balanza a la viabilidad favorable de esta propuesta. Por otro lado, la propia Universidad se beneficiaría fortaleciendo su imagen frente a la sociedad, mejorar su sistema educativo, de investigación e innovación al interactuar con la sociedad en la búsqueda de soluciones. Siendo de interés promover la cultura del emprendimiento por parte del Estado, organizaciones Estatales tales como el Ministerio de la Producción organiza convocatorias para el desarrollo de este tipo de proyectos de apoyo al emprendimiento; el Centro para Emprendedores después de crearse puede participar en este tipo de convocatorias y conseguir el financiamiento para desarrollar su labor de manera más efectiva.

\section{CONCLUSIONES}

De los resultados mostrados, de su análisis y de su discusión, en relación al Diseño de un Centro para Emprendedores en una Escuela Profesional de Ingeniería aplicando el modelo Lean Canvas se pueden obtener las siguientes conclusiones:

1) El análisis interno y externo en relación al emprendimiento empresarial son favorables y sustentan la creación de un Centro para Emprendedores.

2) El perfil emprendedor de los estudiantes muestra un potencial emprendedor, sin embargo, los estudiantes valoran que la creación de una empresa demanda vencer una serie de obstáculos y es necesario entre otros estar capacitado; el Centro de Emprendedores brindaría apoyo en su formación y en la creación de empresas.

3) Los estudiantes consideran que la Universidad debería entre otros formar a los estudiantes en la creación de empresas y desarrollar diversos programas para sacar adelante proyectos emprendedores e innovadores, brindar apoyo para crear modelos de negocios y asesoría personalizada, estas demandas son tomadas en cuenta en los objetivos a alcanzar por el Centro de Emprendedores propuesto.

4) La aplicación del modelo Lean Canvas facilito el desarrollo del proyecto de diseño tomando en cuenta que los conocimientos de las necesidades del cliente son importantes para determinar sus problemas y plantear soluciones, establecer los canales, flujos de ingresos, estructura de costos entre otros,

5) El Plan de Emprendimiento propuesto considera diversas actividades a desarrollar siendo económicamente viable y necesario para los estudiantes en su formación y capacitación.

\section{AGRADECIMIENTOS}

Al Master en Gestión de la Ciencia y la Innovación de la Universidad Politécnica de Valencia por la formación recibida. 


\section{REFERENCIAS}

Aguado, Ma., La Guía del emprendedor de la Idea a la Empresa, 245 p, AEJV Bancaja, Edita: Asociación de Jóvenes Empresarios de Valencia España, (2008)

Ajzen, I., The theory of planned behavior, doi: 10.1016/0749-5978(91)90020-T Organizational Behavior and Human Decision Processes, 50(2), 179-211, (1991)

Arroyo, M. y van der Sijde., Entrepreneurship encouragement and business development support at universities and science parks proposal for a new conceptualization, Industry and Higher Education, 22 (1), 37-48, (2008)

Arroyo, M., Emprendimiento y Universidad Emprendedora: Conceptualización, propuesta metodológica y caracterización de la Universidad Politécnica de Valencia. Tesis Doctoral, Universidad Politécnica de Valencia (2016).

Castro, E. y I. Fernández de Lucio., Innovación y Sistemas de Innovación, 87 p., Valencia, España, (2001)

García del Junco, J., P. Álvarez y R. Reyna., Características del Emprendedor de Éxito en la Creación de PYMES españolas, Estudios de Economía Aplicada, 25 (3), 951-974.Asociación Internacional de Economía Aplicada, Valladolid, España (2007)

Gibb, A., G.Haskins., P. Hannon y I. Robertson., Leading the entrepreneurial University, NCEE, Said Business School, University Of Oxoford, 54 p., (2012)

Gonzales de Olarte, E., La necesidad de una Política de Educación Superior a largo plazo en el Perú, en Aproximaciones de la Educación Universitaria-Perú, Pontificia Universidad Católica del Perú, ISBN: 31501361600477, Lima, Perú, (2016)

Gonzales, J. y C. López., El emprendimiento en los sistemas universitarios, ISBN: 978-980-6810-67-9 y ISBN Volumen: 978-980-6810-74-7, El Tecnológico de Monterrey. Serie Políticas Públicas y Transformación Productiva, No 4., Corporación Andina de Fomento, (2012)

Guerrero, M. y D. Urbano., The creation and development of entrepreneurial universities in Spain. Nova Science Publishers, Inc. (2011)

Guerrero, M., D. Urbano y otros cuatro autores., Observatorio del Emprendimiento Universitario en España. Edición 20152016, Madrid: Crue Universidades Españolas-Red Emprendía -CISE (2016)

Hayter, C.S., A.J. Nelson., S. Zayed, y A. C. O'Connor., Conceptualizing academic entrepreneurship ecosystems: a review, analysis and extension of the literature, doi.org/10.1007/s10961-018-9657-5, J Technol Transf, 43:1039-1082 (2018).

Huang-Saad, A. y S. Celis., How Student Characteristics Shape Engineering Path ways to Entrepreneurship Education, International Journal of Engineering Education, 33 (2), pp. 527-537, (2017)

Liñan, F.,y Y.W. Chen., Development and Cross-Cultural application of a specific instrument to measure entrepreneurial intentions. Entrepreneurship theory and practice, 33(3), 593-617, (2009)

Liventsova, EY. y I.Y Malkova., Development of social and entrepreneurial competency of students within the educational process of a classical university Tomsk State University Journal, No 425, paginas 187-190, (2017)

Marchioni, J., Las Métricas claves del Lean Canvas. XXXVI Congreso del Instituto Argentino de Profesores Universitarios de Costos, La Pampa, Argentina, (2013)

Maurya, A., Running Lean, Iterate from Plan A to a Plan that works, O Reilly, Eric Ries, Series Editor (2012)

Mendoza, J., Y. García y G. Gutiérrez., Iniciativa emprendedora de los estudiantes de una Institución Superior del Estado de Hidalgo. XXI Congreso Internacional de Contaduría, Administración e Informática, México (2016)

Miranda, F.J., A. Chamorro-Mera y S. Rubio., Academic entrepreneurship in Spanish universities: An analysis of the determinants of entrepreneurial intention, doi.org/10.1016/j.iedeen.2017.01.001 European, Research on Management and Business Economics 23: 113-122 (2017)

Observatorio Ocupacional, Manual para la creación de empresas por universitarios, ISBN, 84931726-1-8, Deposito Legal A-210-2002, Quinta impresión, Universidad Miguel Hernández de Elche (2002)

Oneill, P., N. Kimberley y C.Wei Teng., Public University Models for education-from innovation to entrepreneurhip, doi.org/10.4995/HEAd17.2017.5281, 3rd International Conference on Higher Education Advances, Universitat Politecnica de Valencia (2017)

Osterwalder,A. y Y. Pigneur., Generación de Modelos de Negocio. Un Manual para visionarios, revolucionarios y retadores, ISBN: 978-84-234-2841-0, Ediciones Deusto, Barcelona, España (2011)

Paños, J., Educación emprendedora y metodologías activas para su fomento, doi.org/10.6018/reifop.20.3.272221 Revista Electrónica Interuniversitaria de Formación del Profesorado, 20(3), 33-48, Universidad de Deusto, Bilbao, España, (2017)

Patrón, P., La Investigación en la Universidad Peruana, en Aproximaciones de la Educación Universitaria-Perú, 2016. ISBN: 31501361600477, Pontificia Universidad Católica del Perú, Lima, Perú, (2016) 
Reynolds, P., N. Bosma y otros cinco autores., Global entrepreneurship monitor: Data collection design and implementation 1998-2003.Small business economics, 24(3), 205-231, (2005)

Sparano, H.. Emprendimiento en América Latina y su impacto en la gestión de proyectos, Dimensión Empresarial, 12(2), 95-106. (2014)

Serida, J., Guerrero, C. y otros tres autores., Global Entrepreneurship Monitor Perú 2016-2017, ISBN 978-612-4110-757, 69 p, Universidad ESAN, Lima, (2017)

Veciana, J.M., M. Aponte y D. Urbano., University students' attitudes towards entrepreneurship: A two countries comparison. The International Entrepreneurship and Management Journal, 1(2), 165-182, (2005)

Vicens,L. y S. Grullon., Innovación y emprendimiento: Un modelo basado en el desarrollo del emprendedor, V Foro de Competitividad de las Américas, Santo Domingo, Rep. Dominicana Octubre 5-7, (2011)

Wang, YN., Y. Wang, Y.Guo y P. liu., Exploration on the Mode of Innovation and Entrepreneurship Education in Commercial Universities, 6th International Conference on Social Science, Education and Humanities. Colección: Advances in Social Science Education and Humanities Research, Volumen: 185, 590-594 (2017) 
